\title{
SPARC-induced migration of glioblastoma cell lines via uPA-uPAR signaling and activation of small GTPase RhoA
}

\author{
SATEESH KUNIGAL ${ }^{1}$, CHRISTOPHER S. GONDI $^{1}$, MEENA GUJRATI $^{2}$, \\ SAJANI S. LAKKA ${ }^{1}$, DZUNG H. DINH ${ }^{3}$, WILLIAM C. OLIVERO ${ }^{3}$ and JASTI S. RAO ${ }^{1,3}$ \\ ${ }^{1}$ Program of Cancer Biology, Department of Cancer Biology and Pharmacology; ${ }^{2}$ Department of Pathology; \\ ${ }^{3}$ Department of Neurosurgery, University of Illinois College of Medicine at Peoria, One Illini Drive, Peoria, IL 61605, USA
}

Received July 3, 2006; Accepted August 29, 2006

\begin{abstract}
Secreted protein acidic and rich in cysteine (SPARC) is highly expressed in human gliomas where it promotes invasion and delays tumor growth, both in vitro and in vivo. SPARC, which interacts at the cell surface, has an impact on intracellular signaling and downstream gene expression changes, which might account for some of its effects on invasion and growth. Additionally in vitro studies demonstrated that SPARC delays growth, increases attachment, and modulates migration of tumor cells in an extracellular matrixspecific and concentration-dependent manner. Because the signaling aspect of this migration is neither well understood nor characterized, we overexpressed SPARC in both the minimally-invasive U87 cell line and in the most aggressive invasive cell line, SNB19. We first performed RT-PCR analysis
\end{abstract}

Correspondence to: Dr J.S. Rao, Program of Cancer Biology, Department of Cancer Biology and Pharmacology, University of Illinois College of Medicine at Peoria, One Illini Drive, Peoria, IL 61605, USA

E-mail: jsrao@uic.edu

Abbreviations: SPARC, secreted protein acidic and rich in cysteine; MMP, matrix metalloproteinase; RNAi, RNA interference; siRNA, short interfering RNA; uPA, urokinase-type plasminogen activator; UPAR, urokinase-type plasminogen activator receptor; CMV, cytomegalovirus; PBS, phosphate-buffered saline; FITC, fluorescein 5-isothiocyanate; EV, empty vector; H\&E, hematoxylin and eosin; GAPDH, glyceraldehyde-3-phosphate dehydrogenase; pU2, plasmid siRNA vector for UPA and uPAR; pGFP, plasmid siRNA for GFP; puPAR, plasmid siRNA vector for uPAR; puPA, plasmid siRNA vector for uPA; PI3-K/Akt, phosphoinositide 3-kinase/serine and threonine kinase; FAK, focal adhesion kinase; RT-PCR, reverse transcriptase polymerase chain reaction; ECM, extracellular matrix; VEGF, vascular endothelial growth factor; SDS-PAGE, sodium dodecyl sulphate-polyacrylamide gel electrophoresis; BSA, bovine serum albumin; GPCR, G-protein coupled receptor; BFGF, basic fibroblast growth factor; TNF- $\alpha$, tumor necrosis factor $\alpha$; ANG-1, angiopoietin-1; HBEGF, heparin-binding EGF-like growth factor; IGF-1, insulin-like growth factor 1

Key words: glioblastoma, SPARC, uPA-uPAR signaling, small GTPase RhoA, migration and observed an upregulation of UPA and its receptor, uPAR. We also observed increased expression levels of matrix metalloproteinase-2 and -9 (MMP-2 and MMP-9). Western blot analysis confirmed these results, and the enzymatic activity of the metalloproteinases and uPA was further supported by zymography. Downstream of the uPA-uPAR interaction, upregulation of PI3-K occurred in cells overexpressing SPARC. Using GST-TRBD, we showed the upregulation of active GTP-bound RhoA, but neither Rac1 nor Cdc42 were activated. The inhibition of UPA and UPAR downregulated PI3-K activity and cell migration, as shown by matrigel invasion assay. A dorsal skin-fold chamber model revealed the high angiogenic activity of SPARC, though the proliferation of SPARC overexpressing cells was unaffected. Our results show that the small GTPase RhoA was a critical mediator of invasion or migration in the uPA-uPAR/PI3-K signaling pathway.

\section{Introduction}

Expressed during many stages of development in a variety of organisms, the matricellular protein SPARC (secreted protein acidic and rich in cysteine, also known as osteonectin or BM40 ) is expressed primarily in tissues that undergo consistent turnover or in sites of injury/disease in adult vertebrates (1). The capacity of SPARC to bind to several resident proteins of the extracellular matrix (ECM), to modulate growth factor efficacy, to affect the expression of matrix metalloproteinases (MMPs), and to alter cell shape as a counter adhesive factor, supports the idea that SPARC acts to regulate cell interaction with the extracellular milieu during development and in response to injury in vivo.

In an attempt to clarify current information and to propose functions for SPARC in living tissues, Golembieski and Rempel (2) confirmed that SPARC was expressed at high levels in neurogliomas and melanomas, respectively. It has been demonstrated that SPARC is present in high levels in Grade 2 and 3 bladder cancers (3), while others have implicated elevated levels of SPARC in renal cell carcinomas with subsequent tumor development and neovascularization and/or invasion (4). Further two groups have highlighted the fact that SPARC is found in high levels in invasive tumor cells $(5,6)$.

The uPA-uPAR complex is a multifunctional system involved in wound healing, immune response, vascular 
remodeling and cancer. Invasion of cancer cells is a complex process in which the tumor cells migrate from the primary site through the ECM barrier into surrounding normal tissue by degrading the ECM. This complex process involves many proteolytic enzymes which are either serine proteases, especially those constituting the plasminogen activator/ plasmin system (7), or matrix metalloproteinases (8). Urokinase-type plasminogen activator (uPA) is often expressed at high levels in malignant tumors (9), and its activity is high and localized at the invasive edge of these tumors (10). A large body of evidence suggests that uPA plays a key role in tumor progression and invasion by virtue of its ability to activate plasminogen, which degrades many ECM components and activates latent collagenases (11). The effects of uPA-uPAR are not restricted to localizing proteolysis on the cell surface, but are mediated by various signaling pathways activated when uPA binds to UPAR, as well as by uPAindependent functions of uPAR. Signaling cascades induced by uPA-uPAR have been widely studied and found to be exclusively cell specific (12).

$\mathrm{PI} 3-\mathrm{K}$ is a heterodimer composed of a regulatory $\mathrm{p} 85$ subunit and a $110-\mathrm{kDa}$ catalytic subunit. The ability of p85 to associate with other proteins links PI3-K to distinct signaling cascades required for proliferation, adhesion, and motility (13). PI3-K has been shown to act downstream of FAK in cell migration and survival. Kusch et al (14) showed that uPA induces PI3-K activation, which has a role in vascular smooth muscle cell migration. PI3-K and small Rho GTPases such as $\mathrm{Cdc} 42$ and Rac1 are key effectors that regulate cell migration by means of dynamic changes in the actin cytoskeleton $(15,16)$. The present study was undertaken to assess the importance of SPARC activation of uPA-uPAR signaling in cell migration via activation of the PI3-K pathway involving the GTPase RhoA. The results of the present study demonstrate an additional function to SPARC: activation of the PI3-K/Akt pathway via the upregulation of uPA-uPAR signaling in tumors.

\section{Materials and methods}

Cell culture and isolation of stable cell line. SNB19 and U87 glioblastoma cells were cultured in advanced DMEM supplemented with $2 \% \mathrm{FBS}$ in a humidified $\mathrm{CO}_{2}$ incubator at $37^{\circ} \mathrm{C}$. SNB19 and U87 cells were transfected with SPARC cDNAcontaining vectors using FuGene 6. Stable transfectants were selected with medium containing $600 \mu \mathrm{g} / \mathrm{ml}$ of G418. Cells transfected with an empty expression vector served as a control. Medium containing $250 \mu \mathrm{g} / \mathrm{ml}$ of $\mathrm{G} 418$ was used to maintain the stable transfectants.

Transfection studies. The cells were transfected in serumfree media using FuGene 6 transfection reagent (Roche, Indianapolis, IN) according to manufacturer's instructions. pSPARC and pEV were used for transfection. pEV, a pcDNA vector carrying a scrambled nucleic acid sequence, served as a secondary control; no plasmid was introduced in the main control plate. For the transfections, SNB19 and U87 cells were cultured in 100-mm dishes until $50 \%$ to $70 \%$ confluence was obtained. Eight to ten $\mu \mathrm{g}$ of plasmid DNA were mixed with FuGene 6 in serum-free medium per manufacturer's instructions, and added to the cells. After allowing $5 \mathrm{~h}$ for optimal transfection, the serum-free medium was replaced by complete, supplemented medium, and cells were incubated for $48 \mathrm{~h}$. Cells were harvested for the collection of total RNA and/or whole cell lysate for RT-PCR or Western blot analysis. For zymography and in vitro angiogenic assays, the complete, supplemented medium was replaced with serum-free medium $36 \mathrm{~h}$ post-transfection. The conditioned media were collected $12 \mathrm{~h}$ later.

Reverse transcription PCR. Total RNA was isolated from the control and transfected cells using TRIzol according to standard protocol. Total RNA was treated with DNase I (Invitrogen, Carlsbad, CA) to remove contaminating genomic DNA. Firststrand cDNA was prepared using Superscript III reverse transcriptase (Invitrogen). First-strand cDNA (100 ng) was used for PCR amplification using the primers for SPARC, UPA, MMP-9 and VEGF. GAPDH was used as an internal control. PCR analysis was performed per standard protocol.

Western blot analysis. SNB19 and U87 cells were transfected with $\mathrm{pEV}$ and pSPARC as described above. At the end of incubation, cells were washed with ice-cold PBS and lysed in RIPA buffer containing protease inhibitors. Whole cell extracts were subjected to SDS-PAGE and were analyzed by the respective antibodies using standard Western blot protocol.

Matrigel invasion assay. The invasiveness of the SNB19 and U87 cells was tested in vitro with the Boyden chamber invasion assay after transfection with either the $\mathrm{pEV}$ or the vector overexpressing SPARC (pSPARC). Transwell inserts with 8- $\mu \mathrm{m}$ pores were coated with $0.7 \mathrm{mg} / \mathrm{ml}$ Matrigel (Collaborative Research, Inc., Boston, MA). SNB19 and U87 cells were detached using $5 \mathrm{mM}$ EDTA, and $500 \mathrm{ml}$ of the cell suspension $\left(1 \times 10^{6}\right.$ cells $\left./ \mathrm{ml}\right)$ was added to the wells. After incubation for $24 \mathrm{~h}$ at $37^{\circ} \mathrm{C}$, the cells were stained with Hema-3. The cells and Matrigel on the top surface of the filter were carefully removed with a cotton swab. The invasive cells adhering to the bottom surface of the filter were quantified under a light microscope (x20). The data are presented as the average number of cells attached to the bottom surface from randomly chosen fields. Experiments were performed in triplicate filters and were counted at five areas.

Immunofluorescence microscopy. We used a previously described protocol (17-19) with minor changes. Briefly, the cells on the cover slips were washed once with PBS after the stated incubation time, and fixed in $4 \%$ paraformaldehyde (w/v) in PBS for 15 min at RT, followed by three PBS washes. Cells were then permeabilized with $0.5 \%$ Triton X-100 (w/v) in PBS for 10 min, followed by three PBS washes. Finally, the cells were blocked with $1 \%$ BSA (w/v) in PBS overnight at $4^{\circ} \mathrm{C}$. For staining, the cells were incubated in uPAR antibody solution. The primary antibodies on the cell were detected by counter-reaction with secondary antibody conjugated with fluorescent materials. For double staining, the cells were also incubated in anti-SPARC primary antibody and the corresponding secondary antibody, as described above, followed by three washes with PBS and mounted onto a glass-slide in Vectashield. The results were documented using a fluorescence microscope. 
Rho protein pull-down assay. GST-TRBD and GST-PAK PBD fusion proteins were expressed in Escherichia coli and affinity purified. Glutathione agarose beads containing 20-30 $\mu \mathrm{g}$ of GST-TRBD or GST-PAK PBD were incubated with lysates of transfected and non-transfected SNB19 and U87 cells for $60 \mathrm{~min}$. The proteins precipitated on the agarose beads were washed three times with wash buffer and then eluted by boiling in loading buffer at $95^{\circ} \mathrm{C}$ for $10 \mathrm{~min}$. The samples were subjected to SDS-PAGE and immunoblotting with corresponding antibodies.

Fibrin zymography. Zymography was used to determine the activity of the plasminogen activators as previously described (20). The samples were subjected to SDS-PAGE with $10 \%$ gels that contained fibrinogen and plasminogen. Following electrophoresis, the gels were washed twice with $2.5 \%(\mathrm{v} / \mathrm{v})$ Triton X-100 for 30 min each time to remove SDS. Finally, the gels were incubated with $0.1 \mathrm{M}$ glycine buffer $(\mathrm{pH}$ 7.5) at $37^{\circ} \mathrm{C}$ overnight, stained with amido black, and then destained. Final gel had a uniform background except in regions to which uPA had migrated and cleaved its substrate.

Gelatin zymography. Gelatin-substrate gel electrophoresis was performed as described previously (21). SNB19 and U87 cells were transfected with pEV or pSPARC. To collect conditioned media, cells were washed once with serum-free medium and incubated with fresh serum-free medium. After 12-14 h, conditioned media were collected, centrifuged to remove cellular debris, and protein concentrations were determined. Equal amounts of protein were subjected to $0.1 \%$ gelatin SDS-PAGE, under non-reducing conditions. Gels were washed in $2.5 \%$ Triton $\mathrm{X}-100$ and incubated overnight in Tris- $\mathrm{CaCl}_{2}$ buffer. The gels were then stained with $0.2 \%$ Coomassie blue for $30 \mathrm{~min}$ and destained in $20 \%$ methanol and $10 \%$ acetic acid. The clear bands represent gelatinase activity.

Dorsal skin-fold chamber model. Athymic nude mice $(\mathrm{n}=12$, $\mathrm{nu} / \mathrm{nu}$, male, 30-35 g) were bred and maintained within a specific pathogen-free environment. At the implantation site for the U87 and SNB19 glioma cells, we used the dorsal skinfold chamber preparation that contains 2 layers of the membrane on both sides of a ring. This model is characterized by both solid and diffuse infiltrative glioma growth patterns and represents a versatile technique for the non-invasive analysis of glioma microcirculation and hemodynamics (6).

\section{Results}

Overexpression of SPARC in the U87 and SNB19-transfected cells. To study the effects of SPARC overexpression, we transfected SNB19 and U87 cell lines with a pcDNA mammalian expression vector harboring SPARC sequences driven by a CMV promoter. Increase in mRNA levels was determined by the semi-quantitative reverse transcription PCR. Total RNA was isolated from the cells $48 \mathrm{~h}$ after transfection, and firststrand cDNA was prepared using oligo-dT primed Superscript III reverse transcriptase. PCR analysis was carried out using the primers for SPARC. Clear amplification was observed in the transfected cells at approximately $440 \mathrm{bp}$ (SPARC) as
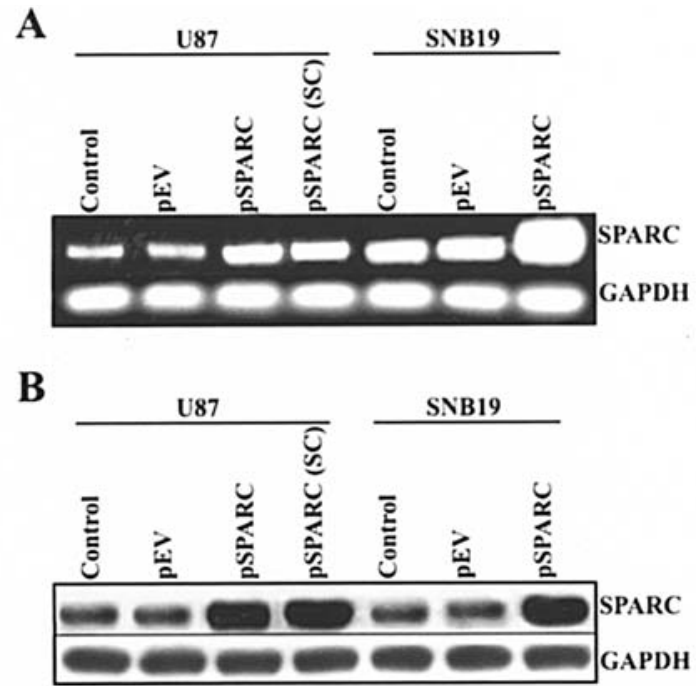

Figure 1. Overexpression of SPARC in the U87 and SNB19 glioblastoma cells. U87 and SNB19 cells were transfected with pSPARC. After $48 \mathrm{~h}$ of incubation, (A) total RNA was extracted using TRIzol reagent, estimated and RT-PCR was performed for assessment of SPARC levels. Expression of GAPDH was verified for the uniform levels of cDNA. (B) SPARC levels were determined at the protein level by Western blot analysis using SPARCspecific antibodies. GAPDH was also immunodetected as a control to confirm equal loading of cell lysates. U87 SPARC (SC), a stable cell line, was included in both the cases in addition to the transfected sample.

compared to non-transfected cells. The levels of GAPDH remained unchanged in all treatment groups, indicating uniform levels of first-strand cDNA taken in all cases (Fig. 1A).

Western blot analysis confirmed that SPARC overexpression increased the levels of SPARC in SNB19 and U87 transfected with pSPARC compared to control and pEV-transfected cells (Fig. 1B).

Levels of MMP-9, $U P A R$ and $U P A$ protein expression. The levels of UPAR protein in the total cell lysate and UPA protein in the conditioned media were assessed by Western blotting and fibrin zymography respectively. Western blot analysis using the anti-uPAR antibody revealed a significant increase in the levels of uPAR after cells were transfected with pSPARC (Fig. 2A).

PCR analysis was performed using the primers for MMP-9, UPAR and uPA. Clear amplification was observed in the transfected cells with pSPARC at approximately $455 \mathrm{bp}$ with the primers for MMP-9, $280 \mathrm{bp}$ with the primers for UPAR and $780 \mathrm{bp}$ with primers for uPA as compared to nontransfected cells. The levels of GAPDH remained unchanged (Fig. 2B).

FACS analysis showed the upregulation of UPAR in the pSPARC-transfected cells as compared to the controls (Fig. 2C). Further immunofluorescence analysis of the localization of UPAR and SPARC revealed that the proteins were co-localized (Fig. 2D). It is well known that UPAR localizes at the leading edge of migrating cells, regardless of their normal or malignant status (Fig. 2D). Gelatin zymography, using conditioned medium from transfected U87 and SNB19 cells with pSPARC increased the levels of MMP-9 when compared to control and pEV-transfected cells (Fig. 2E). 
A

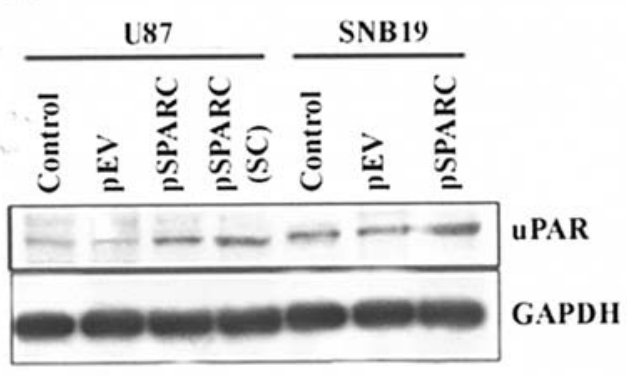

C
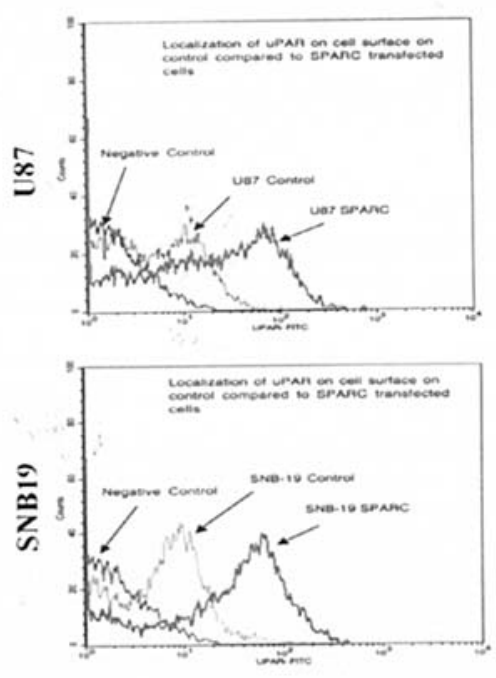

E

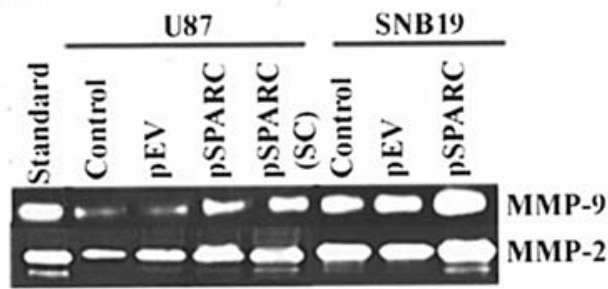

B

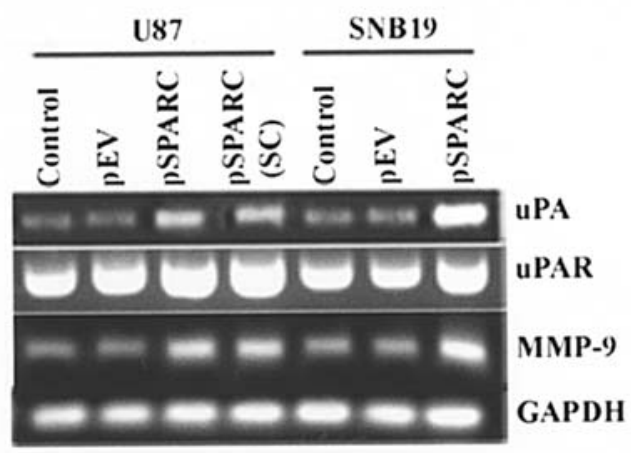

D

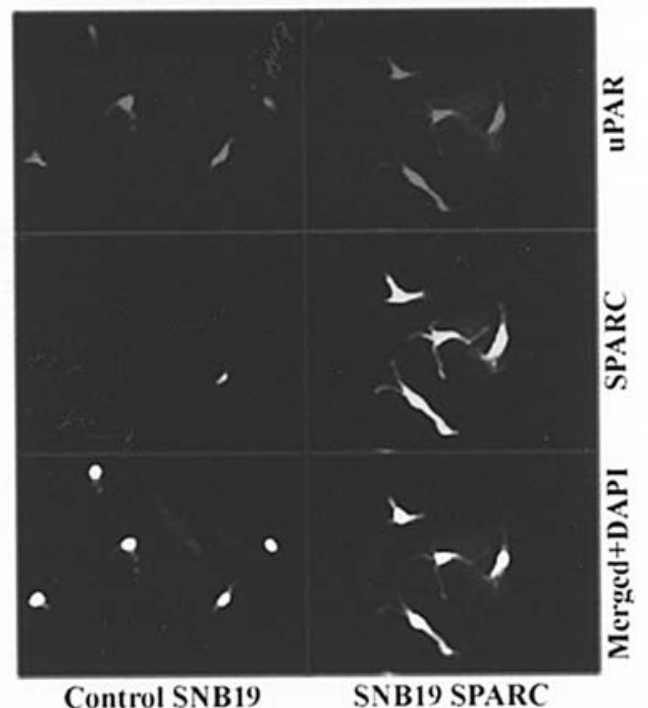

F

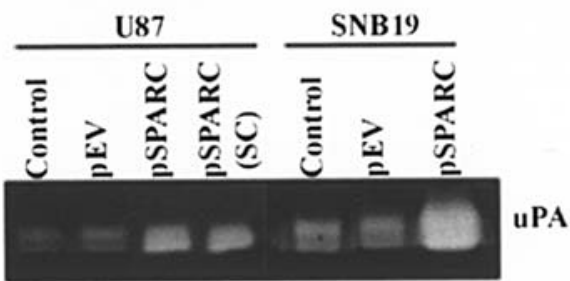

Figure 2. Overexpression of SPARC increased expression of MMP-9, uPA and uPAR. U87 and SNB19 cells were transfected with pSPARC. After 48 h of incubation, (A) uPAR levels were determined at the protein level by Western blot analysis. GAPDH was also immunodetected as control to confirm equal loading of cell lysates. (B) Total RNA was extracted using TRIzol reagent, quantitated and RT-PCR was performed for assessment of uPA, uPAR and MMP-9 levels. Expression of GAPDH was verified for the uniform levels of cDNA. (C) After $48 \mathrm{~h}$ of incubation the cells were subjected to FACS analysis for uPAR expression. (D) Immunocytochemical analysis for the localization of uPAR and SPARC. (E and F) Activities of uPA and MMPs were analyzed by gelatin and fibrin zymography, respectively, by loading equal amounts of protein from the conditioned medium. U87 SPARC (SC), a stable cell line, was included in both the cases in addition to the transfected sample.

Fibrin zymography, using conditioned medium from treated cells, revealed an increase in the levels of uPA activity in the pSPARC-treated cells as compared to the controls. No significant change was observed in the cells treated with the empty vector (Fig. 2F).

Overexpression of SPARC upregulates the invasive potential of SNB19 and U87 cells. The uPA-uPAR system is known to aid in normal cell migration as well as to promote invasion of tumor cells. To study the effect of SPARC-mediated increases of UPA and uPAR and their invasive potential, SNB19 and U87 were transfected with pSPARC and transferred to matrigelcoated transwell chambers and allowed to invade through the matrigel. Staining of pSPARC transfected cells that invaded through the matrigel was much higher compared to the cells transfected with $\mathrm{pEV}$ or control (Fig. 3A). Quantitative analysis of the number of cells showed a two- to three-fold increase in SPARC-transfected cells compared to controls (Fig. 3B). The effect was higher in U87 cells compared to SNB19 cells.

Overexpression of SPARC causes rapid and transient activation of RhoA, but not Racl or Cdc42. We addressed the question 


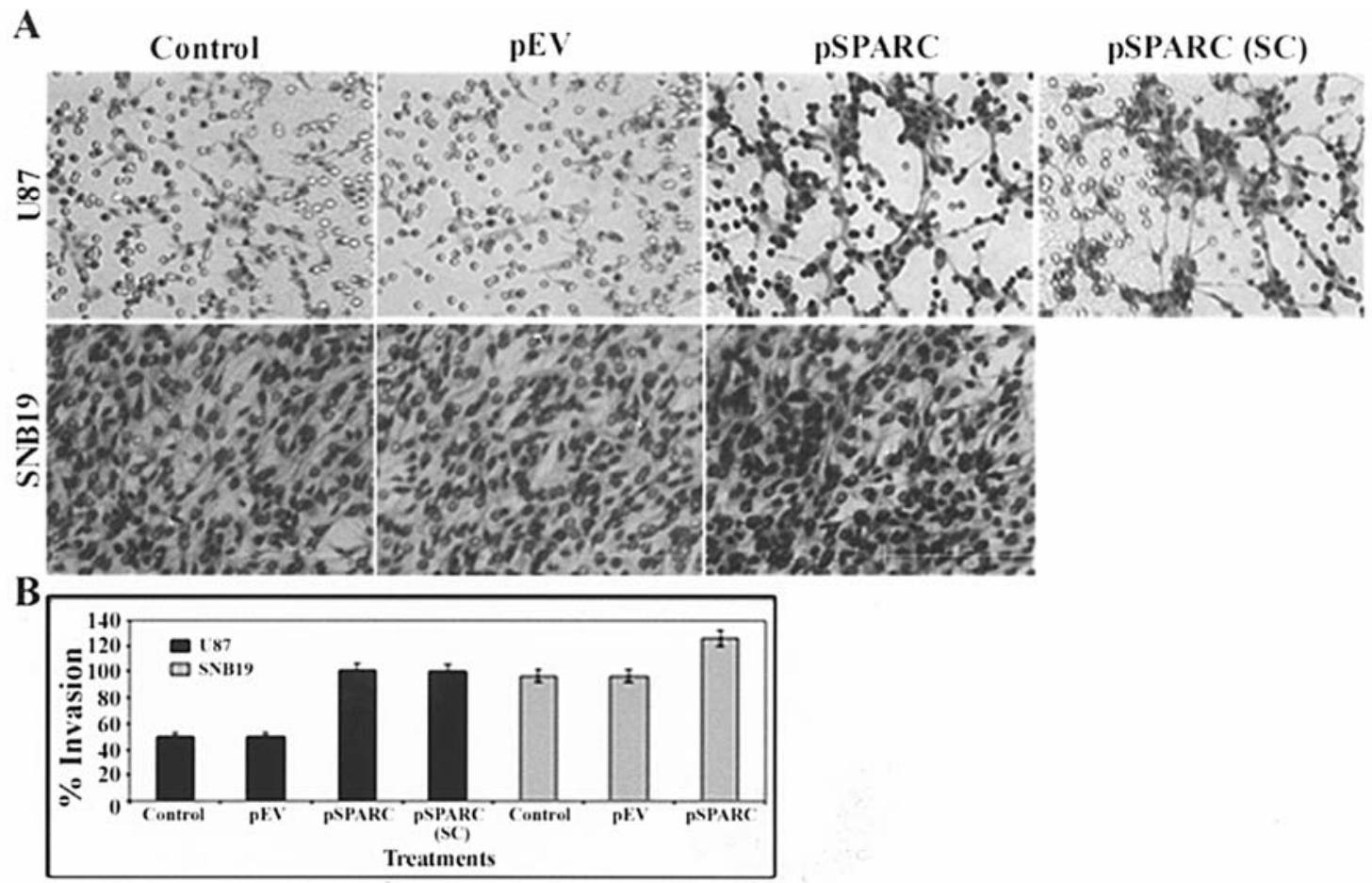

Figure 3. Overexpression of SPARC upregulates the invasive potential of SNB19 and U87 cells. U87 and SNB19 cells were transfected with pSPARC and incubated for $48 \mathrm{~h}$. After the incubation period, the cells were trypsinized and washed once with serum-free medium. Cells $\left(1 \times 10^{6}\right)$ from each treatment were allowed to migrate through matrigel-coated transwell inserts ( $8-\mu \mathrm{m}$ pores) for $24 \mathrm{~h}$. The cells that invaded through the matrigel-coated inserts were stained (A) and photographed under a light microscope at x20 magnification and the percentage of invasion quantitated. (B) As described in the Materials and methods. Shown are the mean \pm SD values from four separate experiments. U87 SPARC (SC), a stable cell line, was included in both cases in addition to the transfected sample.

of whether or not RhoA, Rac1 and Cdc42 are activated in response to SPARC overexpression using pull-down assays. For Rho proteins, both the TRBD-containing and the PAKPBD-containing GST fusion proteins were employed. The lysates of control and SPARC-overexpressing cells were incubated with the appropriate beads and the precipitated proteins were analyzed by immunoblotting with anti-RhoA, anti-Rac1 or anti-Cdc42 antibodies. As shown in Fig. 4A overexpression of SPARC led to rapid and significant activation of RhoA. In pull-down experiments performed to evaluate the activation of Rac1 and Cdc42 proteins, we observed no significant changes in the amount of active Rac1 and Cdc42, respectively (Fig. 4B and C). The lower panel in the figure demonstrates that equal cell lysate protein amounts were used for precipitation reactions. In various cell types, RhoA and Rac1 might be activated to different stimuli. PI3-K was shown to be acting upstream of Rho proteins.

Overexpression of SPARC does not affect proliferation. The MTT assay is used for the quantitative determination of cellular proliferation and activation. We performed the assay with pSPARC-transfected cells from the U87 and SNB19 lines. We observed no significant effect on cell proliferation from SPARC overexpression (Fig. 5). An earlier study by Rempel (22) also supports these findings.

Overexpression of SPARC enhances angiogenesis in gliomas. A strong line of experimental evidence supports the concept that solid glioma growth is critically dependent on angiogenesis, i.e., the formation of new blood vessels that guarantee tissues sufficient oxygen and nutritional supply (23).
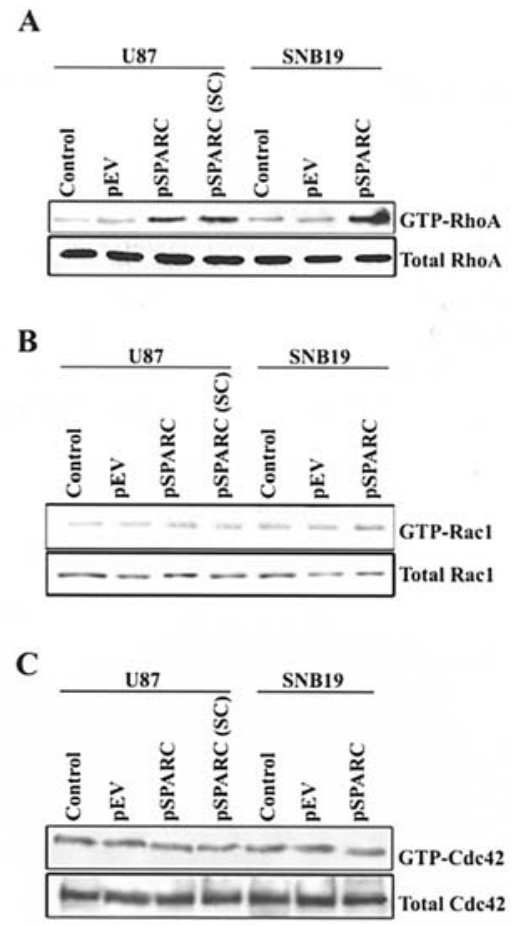

Figure 4. Activation of RhoA but not Rac1 by SPARC. U87 and SNB19 cells were transfected with pSPARC. After a 48-h incubation period, cell lysates were incubated with the GST-TRBD and GST-PAK PBD beads and incubated for 45-60 $\mathrm{min}$. The beads were then washed three times, resuspended in SDS loading buffer, and boiled for $10 \mathrm{~min}$. SDS-PAGE and Western blot analyses were performed. (A) Active RhoA; lower panel, the total RhoA in the control and treatments. (B) Active Rac1 and the total Rac1 in the control and treatments. (C) Active $\mathrm{Cdc} 42$ and total $\mathrm{Cdc} 42$ in the control and treatments. U87 SPARC (SC), a stable cell line, was included in both the cases in addition to the transfected sample. 


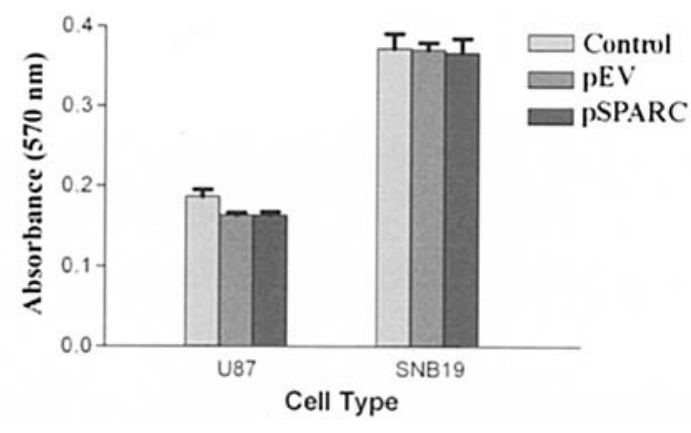

Figure 5. SPARC has no effect on proliferation. U87 and SNB19 cells $\left(1-2 \times 10^{4}\right)$ were plated in microtiter plates, transfected with pSPARC, and incubated for $48 \mathrm{~h}$. Then medium was removed from each well and $20 \mu \mathrm{l}$ of $0.5 \mathrm{mg} / \mathrm{ml}$ MTT (3-4,5-dimethylthiazol-2-yl)-2,5-diphenyltetrazolium) in PBS was added to each well and allowed to incubate for a further $4 \mathrm{~h}$. DMSO $(100 \mu 1)$ was then added to each well to dissolve the formazan crystals. Absorbance values at $550 \mathrm{~nm}$ were measured with a microplate reader. These results were presented as percentage of cells treated with vehicle DMSO $(0.25 \%)$. Shown are the \pm SD values from four separate experiments $(\mathrm{p}<0.05)$.
Implantation of SPARC-overexpressed U87 and SNB19 glioma cells into the dorsal skin-fold chamber induced a strong angiogenic response as compared to the control glioma cells (Fig. 6A). Between the two cell lines, angiogenic activity was higher in SNB19 cells than in U87 cells. The tumor microvasculature demonstrated chaotic micro-angioarchitecture and enormous amounts of new blood vessel formation, which has a 'curly' appearance compared to the pre-existing blood vessel. Our results are in accordance with the findings of a study by Rempel (22), in which the results demonstrated the angiogenic effect of SPARC in C6 rat gliomas.

Further, we demonstrated the involvement of the uPAuPAR system in the SPARC-mediated invasion/migration or angiogenesis. We addressed this question by downregulating uPA (puPA), uPAR (puPAR) and both (pU2) using the siRNA constructs in the SPARC overexpressed SNB19 stable cell line. We performed Western immunoblot analysis using the whole cell extracts from these transfected cells and probed

$\mathbf{A}$

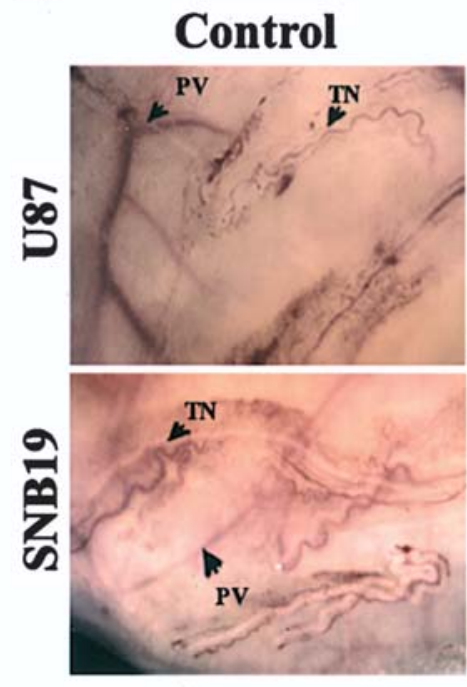

B

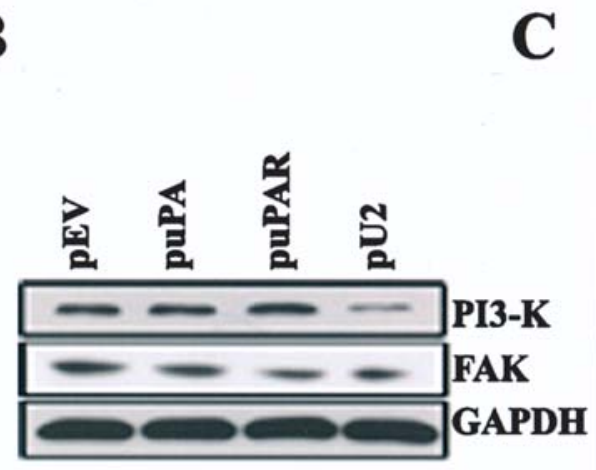

pEV

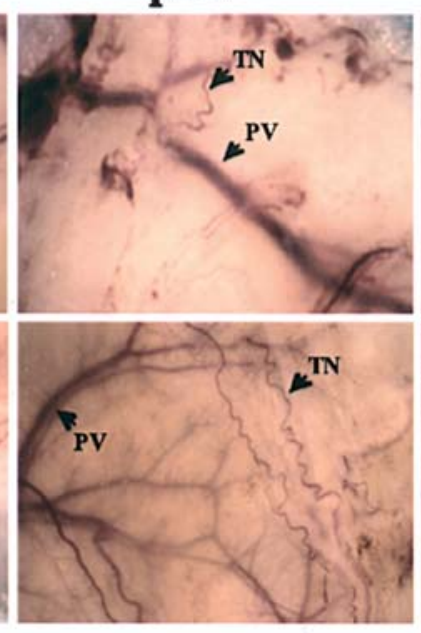

C

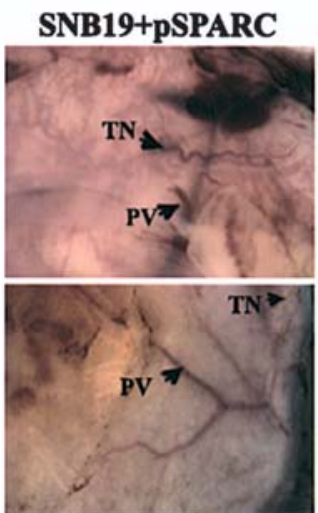

SNB19+pSPARC

+ puPAR
pSPARC
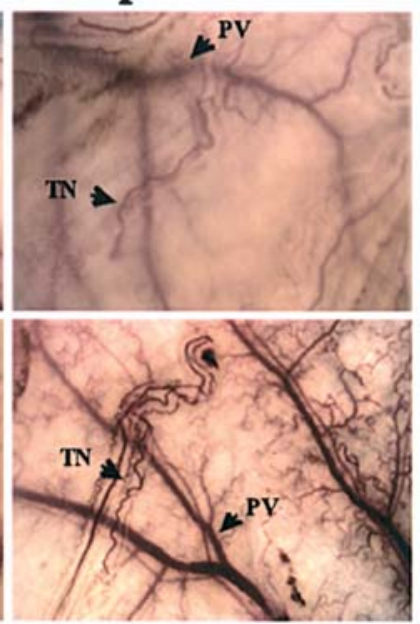

SNB19+pSPARC

+ puPA

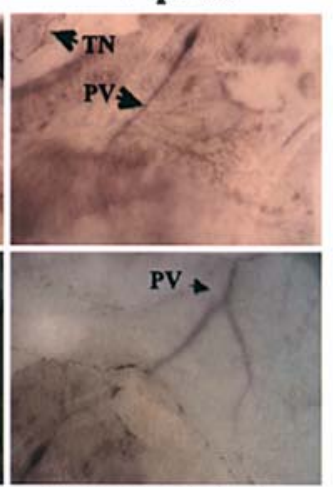

SNB19+pSPARC

+ pU2

Figure 6. Overexpession of SPARC enhances angiogenesis in gliomas. Enhancement of tumor angiogenesis in pSPARC vector was demonstrated by the mouse dorsal window assay. (A) U87 and SNB19 cells were transfected sith pSPARC and incubated for $48 \mathrm{~h}$. After the incubation period, $1 \mathrm{x} 10^{6}$ cells from each treatment condition were suspended in $100 \mu 1$ of PBS, injected into diffuse chambers, and placed under the dorsal skin cavity in nude mice for 10 days. Then, the dorsal skin at the site of the diffuse chamber was removed, observed for neovascularization, and photographed. (B) Involvement of the uPA-uPAR system in the signaling cascade SNB19 SPARC stable cell line was transfected with puPA, puPAR and pU2 and the levels of PI3-K, FAK and GAPDH determined by Western blotting. (C) Inhibition of tumor angiogenesis in SNB19 SPARC stable cell line transfected with various constructs as shown by mouse dorsal window assay. PV, pre-existing vasculature; TN, tumor-induced vasculature. 
with PI3-K and FAK, which are downstream of uPA-uPAR signal transduction pathway, and found that in the cells transfected with biscistronic constructs, there was a considerable amount of reduction in both PI3-K and FAK compared to the pEV-, puPA- and puPAR-transfected cells (Fig. 6B). Fig. 6C shows in vivo angiogenic assay results using the dorsal skin-fold chamber which support the above finding by no neovascularization in the cells transfected with the bicistronic constructs.

\section{Discussion}

In this study, we suggest that SPARC functionally contributes to glioma invasion through the modulation of several mechanisms that regulate tumor growth and migration. It has been reported that SPARC expression is high in cancer cells relative to normal cells, and also that the aggressiveness and migratory capacity of the cancer cells depends on the concentration of SPARC in the ECM. As a result of these experiments, we propose that SPARC is a target candidate for the design of therapies to specifically treat glioma invasion.

Our immunocytochemistry studies as well as earlier reports (24) support the contention that the majority of SPARC is localized to the cytoplasm. However, we cannot rule out the possibility that a small, immunohistochemically undetectable amount of SPARC may be localized to the nucleus. A recent report demonstrated that exogenous SPARC was taken up into endothelial cells and translocated into the nucleus (25) where it could potentially exert its effect on cell division. Thus, we need to consider that SPARC's effects on gliomas may occur both extracellularly and intracellularly. Experiments are currently underway to determine whether SPARC functions via independent mechanisms that affect cell motility and proliferation. In our immunocytochemical analysis, we found that SPARC-transfected cells showed co-localization of UPAR and SPARC. In addition, after probing the cells with anti-uPAR antibody, FACS analysis demonstrated the upregulation of UPAR in SPARC-transfected cells as compared to control and $\mathrm{pEV}$-transfected cells. It has been reported by many researchers that UPAR localizes at the leading edge of migrating cells. This may be one explanation for the high invasive capacity of cells with elevated SPARC expression.

The degradation of existing extracellular matrix (ECM) involves proteolytic enzymes such as plasminogen activators (e.g., uPA) and matrix metalloproteinases (MMPs) and their respective inhibitors (e.g., PAI-1 and TIMP-1) (26-28). The link between uPA-uPAR has long been established (29). The UPA-UPAR system regulates the migration of various cell types and has an important role in the progression of cancer. Our RT-PCR result confirms the upregulation of uPA and its receptor at the mRNA level, supporting earlier reports that the cancer cells overexpress UPA and uPAR (30). In addition, the FACS analysis shows the upregulation of UPAR in SPARC clones and pSPARC-transfected cells. However, we suspect that MMP-2 and MMP-9 activation may, in part, contribute to SPARC's promotion of invasion in gliomas. It has been shown by cDNA array analysis that MMP-2 expression can be upregulated by SPARC (2). Further, SPARC has been shown to induce the activation of MMP-2 in breast cancer cell lines (31). Zymography results revealed
MMP-2 activation in the SPARC-transfected and stable clones as compared to the controls, therefore supporting previously reported data. In our studies, we also found that SPARC clones and transfected cells showed a high content of PI3-K. Previous reports of PI3-K involvement in UPAR signaling may explain the results observed in the present study; PI3-K may also be involved in the activation of Rho GTPases.

We demonstrate here that the small GTPase RhoA is involved in the SPARC-induced migration of SNB19 and U87 cells. RhoA was highly activated in response to SPARC, as demonstrated by the GST-TRBD pull-down assay. Once the G-protein coupled receptors (GPCR) are activated, the Rho proteins are also activated. Because uPAR lacks a transmembrane domain, it requires some additional protein to transduce extracellular stimuli into the cell. The adaptor molecule that is best known in UPAR signal transduction is the integrin $\alpha_{V} \beta_{3}$. Here, the integrin $\alpha_{V} \beta_{3}$ may also be required to transduce the signal to the interior of the cell. Recently, small GTPases, particularly RhoA and Rac1, were shown to be upregulated and exhibited high activity in isolated growing lamellipodia (32). We did not find any change in the amount of GTP-Rac1 and GTP-Cdc42 (data not shown) in our pull-down assays. A possible explanation is that the activation and deactivation of these molecules or redistribution of the active form of the protein may occur in a negligible amount as compared to RhoA. Additionally, redistribution of $\mathrm{Cdc} 42$ to the leading edge of the migrating cells was not observed (32).

Taken together, these results clearly support our hypothesis. Unlike normal cells in culture that express SPARC yet are adherent, U87 cells are weakly adherent and show low to intermediate levels of SPARC. Highly aggressive SNB19 cells show more SPARC expression. SPARC transfection induced intermediate to high adherence in our study. However, exceptionally high levels of SPARC can promote de-adhesion and, therefore, can also induce an intermediate level of adherence $(22,33)$. This represents the contradictory situation that both low and high levels of SPARC can promote more aggressive invasion than intermediate levels.

SPARC may contribute to mechanisms involving uPAUPAR in cell migration. SPARC binds to the inhibitor of UPA (34), and thus, could indirectly promote tumor infiltration by permitting uPA greater receptor interaction, leading to downstream signaling involving PI3-K and small GTPases. Also, its interaction with the ECM molecule vitronectin (34), which is found in high abundance along vessel basement membranes, may directly contribute to tumor invasiveness by facilitating migration along those structures.

In terms of the function of SPARC, we have characterized two aspects: the proliferation and angiogenesis of glioma cells. Proliferation in the overexpressed SPARC cells was not affected when compared to the controls, as shown by the MTT assay. During angiogenesis, endothelial cells proliferate and differentiate under stimulation from various factors such as basic fibroblast growth factor (bFGF), tumor necrosis factor (TNF- $\alpha$ ), angiopoietin-1 (ANG-1), vascular endothelial growth factor (VEGF), heparin-binding EGF-like growth factor (HBEGF), and insulin-like growth factor 1 and 2 (IGF-1 and -2). These angiogenic factors (IGF-2, VEGF, BFGF, 
TNF- $\alpha$ and ANG-1) were significantly increased during the progression of gliomas.

Our dorsal skin-fold chamber implants showed increased neovascularization in SPARC-overexpressed clones. As expected, there was also increased metalloproteinase and urokinase plasminogen activator expression in the tumors. Determining the relative influence of these angiogenic regulators will require functional inhibition studies, which are underway.

In this study, SPARC-mediated invasion of the tumors involved the small GTPase RhoA, the upstream effector to the RhoA at the cell surface is uPA-uPAR. To confirm this, we performed experiments where we downregulated uPA-uPAR, using both single and bicistronic siRNA constructs. Western blot analysis for PI3-K and FAK indicated downregulation of the proteins, most prominently in the bicistronic constructs where we reduced the levels of both UPA and UPAR. This finding suggests the involvement of the uPA-uPAR in the SPARC-mediated invasion/migration of gliomas.

In conclusion, our data highlight that tumor invasion and SPARC overexpression are closely associated and involve PI3-K and RhoA GTPase. These results shed light on potential targets for inhibiting tumor cell infiltration in gliomas and, possibly, other cancers.

\section{Acknowledgements}

We thank Dr Martin A. Schwartz, Cardiovascular Reseach Center, Mellon Prostate Cancer Institute, Departments of Microbiology and Biomedical Engineering, University of Virginia, Charlottesville, VA for affording the plasmid expressing the GST-TRBD fusion protein, Shellee Abraham for preparing the manuscript and Diana Meister and Sushma Jasti for reviewing the manuscript. This study was supported by National Cancer Institute grant CA 75557, CA 92393, CA 95058, CA 116708 and N.I.N.D.S. NS47699 and Caterpillar, Inc., OSF Saint Francis, Inc., Peoria, IL (to J.S.R.).

\section{References}

1. Brekken RA and Sage EH: SPARC, a matricellular protein: at the crossroads of cell-matrix. Matrix Biol 19: 569-580, 2000.

2. Golembieski WA and Rempel SA: cDNA array analysis of SPARC-modulated changes in glioma gene expression. J Neurooncol 60: 213-226, 2002.

3. Yamanaka M, Kanda K, Li NC, Fukumori T, Oka N, Kanayama HO and Kagawa S: Analysis of the gene expression of SPARC and its prognostic value for bladder cancer. J Urol 166: 2495-2499, 2001

4. Sakai N, Baba M, Nagasima Y, Kato Y, Hirai K, Kondo K, Kobayashi K, Yoshida M, Kaneko S, Kishida T, Kawakami S, Hosaka M, Inayama Y and Yao M: SPARC expression in primary human renal cell carcinoma: upregulation of SPARC in sarcomatoid renal carcinoma. Hum Pathol 32: 1064-1070, 2001.

5. Paley PJ, Goff BA, Gown AM, Greer BE and Sage EH: Alterations in SPARC and VEGF immunoreactivity in epithelial ovarian cancer. Gynecol Oncol 78: 336-341, 2000.

6. Vajkoczy P, Menger MD, Goldbrunner R, Ge S, Fong TA, Vollmar B, Schilling L, Ullrich A, Hirth KP, Tonn JC, Schmiedek P and Rempel SA: Targeting angiogenesis inhibits tumor infiltration and expression of the pro-invasive protein SPARC. Int J Cancer 87: 261-268, 2000.

7. Vassalli JD, Sappino AP and Belin D: The plasminogen activator/plasmin system. J Clin Invest 88: 1067-1072, 1991.

8. Matrisian LM: The matrix-degrading metalloproteinases. Bioessays 14: 455-463, 1992.
9. Yamamoto M, Sawaya R, Mohanam S, Bindal AK, Bruner JM, Oka K, Rao VH, Tomonaga M, Nicolson GL and Rao JS: Expression and localization of urokinase-type plasminogen activator in human astrocytomas in vivo. Cancer Res 54: 3656-3661, 1994.

10. Pyke C, Kristensen P, Ralfkiaer E, Grondahl-Hansen J, Eriksen J, Blasi F and Dano K: Urokinase-type plasminogen activator is expressed in stromal cells and its receptor in cancer cells at invasive foci in human colon adenocarcinomas. Am J Pathol 138: 1059-1067, 1991.

11. Dano K, Behrendt N, Brunner N, Ellis V, Ploug M and Pyke C: The urokinse receptor. Protein structure and role in plasminogen activation and cancer invasion. Fibrinolysis 8: 189-203, 1994.

12. Blasi $F$ and Carmeliet P: uPAR: a versatile signalling orchestrator. Nat Rev Mol Cell Biol 3: 932-943, 2002.

13. Vanhaesebroeck B and Waterfield MD: Signaling by distinct classes of phosphoinositide 3-kinases. Exp Cell Res 253: 239-254, 1999.

14. Kusch A, Tkachuk S, Haller H, Dietz R, Gulba DC, Lipp M and Dumler I: Urokinase stimulates human vascular smooth muscle cell migration via a phosphatidylinositol 3-kinase-Tyk2 interaction. J Biol Chem 275: 39466-39473, 2000.

15. Jimenez C, Portela RA, Mellado M, Rodriguez-Frade JM, Collard J, Serrano A, Martinez A, Avila J and Carrera AC: Role of the PI3K regulatory subunit in the control of actin organization and cell migration. J Cell Biol 151: 249-262, 2000.

16. Sturge J, Hamelin J and Jones GE: N-WASP activation by a beta1-integrin-dependent mechanism supports PI3K-independent chemotaxis stimulated by urokinase-type plasminogen activator. J Cell Sci 115: 699-711, 2002.

17. Dumler I, Weis A, Mayboroda OA, Maasch C, Jerke U, Haller H and Gulba DC: The Jak/Stat pathway and urokinase receptor signaling in human aortic vascular smooth muscle cells. J Biol Chem 273: 315-321, 1998.

18. Dumler I, Stepanova V, Jerke U, Mayboroda OA, Vogel F, Bouvet P, Tkachuk V, Haller H and Gulba DC: Urokinaseinduced mitogenesis is mediated by casein kinase 2 and nucleolin. Curr Biol 9: 1468-1476, 1999.

19. Dumler I, Kopmann A, Weis A, Mayboroda OA, Wagner K, Gulba DC and Haller H: Urokinase activates the Jak/Stat signal transduction pathway in human vascular endothelial cells. Arterioscler Thromb Vasc Biol 19: 290-297, 1999.

20. Mohanam S, Jasti SL, Kondraganti SR, Chandrasekar N, Kin Y, Fuller GN, Lakka SS, Kyritsis AP, Dinh DH, Olivero WC, Gujrati M, Yung WK and Rao JS: Stable transfection of urokinase-type plasminogen activator antisense construct modulates invasion of human glioblastoma cells. Clin Cancer Res 7: 2519-2526, 2001.

21. Lakka SS, Jasti SL, Kyritsis AP, Yung WK, Ali-Osman F, Nicolson GL and Rao JS: Regulation of MMP-9 (type IV collagenase) production and invasiveness in gliomas by the extracellular signal-regulated kinase and jun amino-terminal kinase signaling cascades. Clin Exp Metastasis 18: 245-252, 2000.

22. Rempel SA, Golembieski WA, Fisher JL, Maile M and Nakeff A: SPARC modulates cell growth, attachment and migration of U87 glioma cells on brain extracellular matrix proteins. J Neurooncol 53: 149-160, 2001.

23. Millauer B, Shawver LK, Plate KH, Risau W and Ullrich A: Glioblastoma growth inhibited in vivo by a dominant-negative Flk-1 mutant. Nature 367: 576-579, 1994.

24. Rempel SA, Golembieski WA, Ge S, Lemke N, Elisevich K, Mikkelsen T and Gutierrez JA: SPARC: a signal of astrocytic neoplastic transformation and reactive response in human primary and xenograft gliomas. J Neuropathol Exp Neurol 57: 1112-1121, 1998.

25. Gooden MD, Vernon RB, Bassuk JA and Sage EH: Cell cycledependent nuclear location of the matricellular protein SPARC: association with the nuclear matrix. J Cell Biochem 74: 152-167, 1999.

26. Liekens S, De Clercq E and Neyts J: Angiogenesis: regulators and clinical applications. Biochem Pharmacol 61: 253-270, 2001.

27. Rice A and Quinn CM: Angiogenesis, thrombospondin, and ductal carcinoma in situ of the breast. J Clin Pathol 55: 569-574, 2002.

28. Sage EH and Vernon RB: Regulation of angiogenesis by extracellular matrix: the growth and the glue. J Hypertens Suppl 12: S145-S152, 1994.

29. Kjoller L: The urokinase plasminogen activator receptor in the regulation of the actin cytoskeleton and cell motility. Biol Chem 383: 5-19, 2002. 
30. Ploug M, Ellis V and Dano K: Ligand interaction between urokinase-type plasminogen activator and its receptor probed with 8-anilino-1-naphthalenesulfonate. Evidence for a hydrophobic binding site exposed only on the intact receptor. Biochemistry 33: 8991-8997, 1994.

31. Gilles C, Bassuk JA, Pulyaeva H, Sage EH, Foidart JM and Thompson EW: SPARC/osteonectin induces matrix metalloproteinase 2 activation in human breast cancer cell lines. Cancer Res 58: 5529-5536, 1998.

32. Cho SY and Klemke RL: Purification of pseudopodia from polarized cells reveals redistribution and activation of Rac through assembly of a CAS/Crk scaffold. J Cell Biol 156: 725-736, 2002.
33. Greenwood JA and Murphy-Ullrich JE: Signaling of de-adhesion in cellular regulation and motility. Microsc Res Tech 43: 420-432, 1998.

34. Rosenblatt S, Bassuk JA, Alpers CE, Sage EH, Timpl R and Preissner KT: Differential modulation of cell adhesion by interaction between adhesive and counter-adhesive proteins: characterization of the binding of vitronectin to osteonectin (BM40, SPARC). Biochem J 324: 311-319, 1997. 